Gynecol Obstet Invest 1994;38:281-282

\title{
Author Index Vol. 38, 1994
}

Abramov, L. 194 Altaras,M. 14, 127 Anastasio, P.S. 266 Andersson, S.-B. 253 Andolf,E. 51 Aono, T. 5 Aravantinos, D. 140 Arduini,D. 177 Arends,J.W. 183 Aytaç, S. 96 Ayuso, M.J. 157 Aznar, J. 157

Bawdon, R.E. 1 Benedetti Panici, P. 213 Berendtsen, H. 104 Bergström,B. 107 Bergström,S. 31,82, 151, 1 < Beyth,Y. 14,113,127 Biondi,C. 100 Blasi,N. 266 Bonati,M. 60 Bremme, K. 145 Bresadola, M. 236 Brugia, M. 134 Bukovsky, 1.217 Burgut,R. 249 Buzzi,M. 100 Carretti,N. 78 Caspi, E. 217 Castro, R.M.F. 18 Cavallaro,R. 100 Cavanna,C. 130 Çetin,T. 249 Chang, T.-C. 36 Chao,A. 36 Check, J.H. 57 Chirivella, M. 157 Chiu, T.-H. 36 Ciavattini, A. 134 Cicinelli, E. 266 Clementi, M. 134 Cobanoglu,Ö. 272 Cohen, I. 113,127 Colonna,L. 130

Conte,M. 213 Cordoba, M. 127 Crosignani, P.G. 70 Cunha,S.P. 18

David, M.P. 194 Degani, S. 41

DeLeo,V. 60 Delgado,I. 227 Demir,C. 249 De Nictolis, M. 134 DePità,O. 236 DeVita,D. 60 Dilorio,R. 236 Dilger,I. 24 Dümen, G. 96 Dudenhausen, J.W. 24, 227 Dunselman, G.A.J. 183 El Maradny, E. 169 Eremita,G.A. 78 España, F. 157 Estellés,A. 157 Evers,J.L.H. 183 Fabbri, G. 60 Facchinetti, F. 122 Farina, A. 100 Fava,M. 122 Fejgin, M. 113 Ferretti, M.E. 100 Folgosa,E. 198 Fracchiolla, N.S. 70 Franca, L.C. 18 Freedman, R.R. 206 Fujimoto, Y. 245 Fujita, T. 5 Fuke, Y. 5 Fung, H.Y.M. 186 Furuhashi, N. 73

Garzetti, G.G. 134 Genazzani, A.D. 60 Genazzani, A.R. 60,122 Giacomello, F. 177 Gilabert,J. 157 Goeij, A.F.P.M. de 183 Gök§in,E. 96 Golan, A. 217 Goldberger, S. 14,113 Goteri,G. 134 Grab,D. 90 Grancha,S. 157 Grella,P. 78 Guariglia, L. 213 Gunnarsson, P.O. 253 Hadas,E. 217 Hagström, U. 82 Haj,M. 54 Haj,N. 54 Halim,A. 169 Halperin,R. 217 Hattori, S.-E. 65 Herman, A. 217 Högberg,U. 107 Holzinger, M. 14 Hosokawa, K. 245 Hsieh,T.-T. 36 Hsu, J.-J. 36 Hütter, W. 90 Hvidman, L. 21 Iioka,H. 173 Imai,S. 5 Iosif,C.S. 51 Ippoliti,F. 236 Ishikawa, H. 65 Isik,A.Z. 272 Jernström, H. 261 Johansson, C.-J. 253 Jörgensen, C. 51

Kanayama, N. 169 Kaul,S. 1 Kawai, M. 65 Kawano,Y. 191 Kawashima, M. 245 Kikkawa, F. 65 Kimura, C. 73 Kimura, H. 73 Kitao,M. 117 Kock,K. 104 Koishi,K. 245 Kojima, M. 65 Kuhn,W. 210 Kulenkampff, D. 210 Ku³/sçu, E. 272 Kutluay,L. 272 Kuzuya, K. 65 Lai,C.-H. 276 Lehoczky, D. 45 Levinsky, R. 41 Libombo,A. 198 Lin,C.-Y. 276 Linden, P.J.Q. van der 183 Loke, D.F.M. 10 Lopez, H.B.B. 104 Lublin, H. 31 Luthman, M. 145 Machungo, F. 151 Maeda,O. 65 Maehara,K. 169 Maietta Latessa, A. 60 Manabe,Y. 163 Manaka,C. 28 Mancuso, S. 213 Marangoni, E. 130 Marinoni, E. 236 Matonti,G. 213 Mayes,M.M. 206 Maymon, R. 14 Menzo,S. 134 Michalas,S. 140 Micheelsen, U. 104 Michelone,G. 130 Micó,J.M. 157 Minakami, H. 28 Minaretzis, D. 140 Miyakawa, I. 191 Mizuno, K. 65 Molin,A. 31 Mori,N. 191 Mori,T. 163 Muttinelli, C. 223 Nagae, H. 73 Nagata,N.Y. 18 Nakayama, M. 5 Neri,A. 70 Neri,I. 122 Neubert,R. 227 Nicola, S. 130 Nicotra,M. 223 Nordle, Ö. 253

Oettinger,M. 54,87 Ohno,Y. 245 Okada,H. 245 Oldani, S. 70 Olsson,H. 261 Omar,M.M. 107 Osmers,R. 210 Ozaki,T. 117 Öztürk,M. 96 
Pajor, A. 45 Pao,C.C. 276

Pareschi, M.C. 100 Parisi,C. 266 Passi,S. 223 Paternoster, D. 78 Pereira,C. 82 Petersen, L.K. 21

Petraglia,F. 60 Peymer, M. 57 Porcelli,B. 78

Rabia,R. 41 Ratnam,S.S. 10 Rinaldo,D. 177 Rizzo,G. 177 Rogers, M.S. 186 Rolfí, G. 223

Romanini, C. 134, 177 Romano, F. 266 Ron-El, R. 217 Roy,A.C. 10 Rydhström,H. 51

Säfwenberg, J. 82 Saha,N. 10 Saito,T. 73 Sakaguchi, M. 163 Sato, I. 28 Sbracia, M. 223

Scambia, G. 213 Schmidt, G 253 Schneider, D. 90 Schwedler,G. 24 Segal, J. 113 Segre,A. 60

Sgherzi,M.R. 60 Shafík,A. 241 Shapiro, I. 41 Sharf,M. 41 Sharony, A. 87 Shasha,S.M. 54

Shulman,A. 14,127 Sobhi,S. 1 Soffer,Y. 217 Soler,A. 151 Sonnino, A. 177 Spinillo,A. 130

Stock, S. 145 Suehara, N. 5

Suganuma, N. 65 Sugimoto, H. 28 Suren,A. 210 Takahashi,K. 117

Takahashi, T. 28 Tamada, T. 28 Tamakoshi, K. 65 Taniyama, K. 191 Tay,J.S.H. 10 Tepper,R.

14,113,127 Terao,T. 169 Terinde,R. 90 Tomoda, Y. 65 Toppare, M.F. 96 Trecca, D. 70 Tsionou, C. 140 Tziortziotis, D. 140

Uchida,A. 117 Uldbjerg,N. 21

Vardar,M.A. 249 Vercellini, P. 70 Vesce,F. 100 Viegas, O.A.C. 10

Wang,C.-Y. 276 Watanabe,Y. 245 Werner, S. 145 Wolf, A. 90 Wolman,I. 194 Wong,F.W.S.

186 Wong,Y.L. 186 Woodward, S. 206

Yajima, A. 73 Yamasaki, H. 117 Yamashita,S. 245 Yarkoni,S. 113 Yoshino,K. 117

Zaccardo, M. 57 Zanconato, G. 151 Zorlu,C.G. 272

282

Author Index 